\title{
Photometry of Variables in Close Visual Double Stars
}

\author{
O. G. FRANZ, R. L. MILLIS, and N. M. WHITE (Flagstaff)
}

\begin{abstract}
A method of photoelectric photometry of the components of visual double stars is briefly described. Observations of ADS $9701=\delta$ Ser and of UV Aur $=$ ADS 3934 B are presented to demonstrate the usefulness of this technique for the accurate photometry of variables in close visual double stars.
\end{abstract}

One of the consequences of duplicity among intrinsic variable stars is that for systems having separations less than 5 arc seconds it is virtually impossible using conventional photoelectric techniques to obtain undiluted light curves or, in many cases, even to tell which component is variable. Yet it is in such systems that one wants to know as much as possible about variable stars since the existence of duplicity may lead to a determination of the luminosity and even the mass of the variable component. The recently developed technique of photoelectric area scanning (RAKOS 1965; FRANZ 1966) now makes it possible to do accurate photometry of the individual components of double stars down to separations as small as 1 arc second. The purpose of this paper is to describe the area scanning technique as it applies to variable stars and to present recent results for two previously inaccessible stars.

A photoelectric area scanning photometer specifically designed for photometry of visual binaries (FRANZ 1970) is shown in Figure 1. This instrument differs from a conventional photometer primarily in that the fixed entrance aperture is replaced by a moving slit. The slit repetitively sweeps at a constant rate across a given area in the focal plane of the telescope. During each sweep the output of the photomultiplier is amplified and accumulated in the memory of a multichannel analyzer. Since the sweep trigger of the multichannel analyzer is synchronized with the slit driving mechanism, it is possible to integrate over any preset number of sweeps.

When using the area scanner for photometry of visual binaries, the slit is scanned across the two components along the line joining them. After a sufficient number of sweeps, a welldefined, double-peaked image profile is obtained. Reduction of such a profile to brightnesses of the individual components consists of determining by iterative least-squares solutions the parameters of two functions whose sum best represents the observed image profile. Although initially Gaussian functions were used, it was subsequently found (FRANZ and CROWFOOT 1971) that a better representation of the image profile is given by functions of the form

$$
\begin{aligned}
& I=\frac{H}{1+\left\{\frac{a b s(x-A)}{B}\right\}^{P}}, \text { where } \\
& P=P_{0}\left\{1+\frac{a b s(x-A)}{C}\right\} .
\end{aligned}
$$

In this expression "I" is the intensity at various positions along the scan, "A" the peak location, "B" the half-power width, and "C" a parameter which determines the rate at which the variable exponent "P“ varies along the scan. The superpriority of this function over a Gaussian is clearly seen in Figure 2. The brightness of the two components are found directly from the areas of the two functions which give the best fit. Because of the small angular separation of the components and the fact that the measurements of each are simultaneous, accurate magnitude differences can be derived from this type of data without correction for atmospheric extinction, transparency variations, or fluctuations in the sensitivity of the equipment.

ADS 9701, perhaps better known as $\delta$ Ser, is well suited to study by the area scanning technique. The two components are Fo subgiants having a brightness difference of about one magnitude and an angular separation of 3.9 arc seconds. Observations made in 1967 with a 

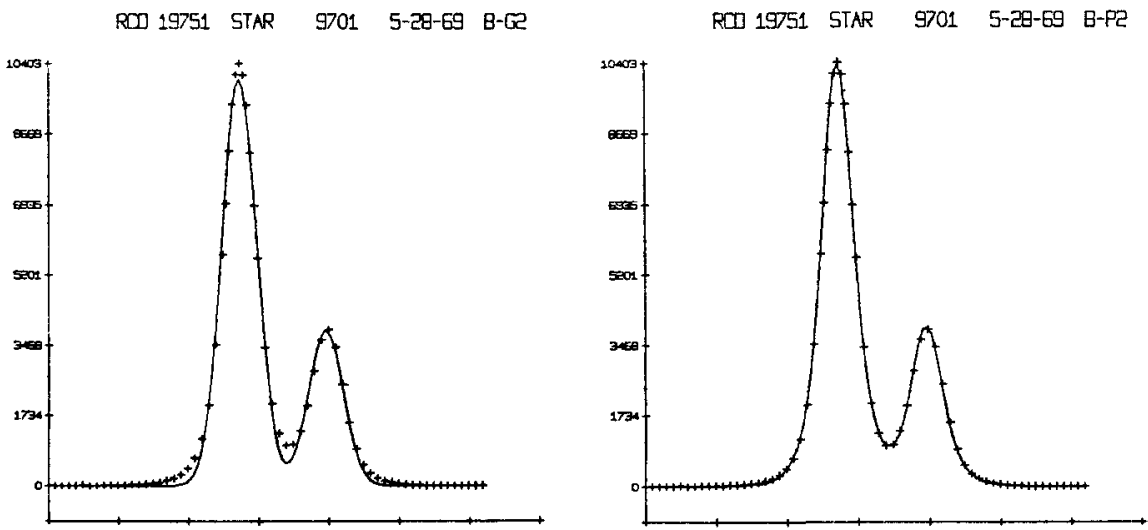

Fig. 2: Comparison of an observed (crosses) and computed (solid lines) image profile of the 3.9-arc-sec binary ADS $9701=\delta$ Ser, the left side showing representation by Gaussian functions, the right side representation by functions of the type described in the text.

conventional photometer (MILLIS 1967) showed that the combined brightness of the two components varied by as much as five-hundredths of a magnitude on a time scale of a few hours. The light curves obtained at that time are characteristic of $\delta$ Sct variables, but because of the similarity in the spectral types of the components it is not possible to decide with certainty on the basis of these observations alone which component is variable.

We have observed $\delta$ Ser extensively with the area scanner since 1969. As is shown in Figure 3, our data demonstrate that all of the variation originates in the brighter component. In this diagram we have plotted the magnitude differences between the two components as well as the instrumental magnitudes of each for two different nights. Figure 4 contains some of our 1971 light curves for $\delta$ Ser. Clearly the variations are not strictly periodic, but most of the well-defined maxima can be fitted approximately by a period of about three hours. It is interesting to note that the 1969 data are in closer agreement with a period near six hours. Also, the average magnitude difference between the two components in blue light was about $0.1 \mathrm{mag}$ greater in 1971 than in 1969 . Combined light photometry shows that this is the result of a systematic brightening of the variable component. The amplitude of brightness variation is strongly modulated ranging from $0.2 \mathrm{mag}$ to less than 0.07 mag. Amplitude modulation of this type is typical of $\delta$ Sct stars.

ADS 3934 is a 3.5 arc second double star whose visual primary is the Mira-type, carbon star UV Aur, a variable with a period of nearly 400 days, and whose secondary component appears to be a late B-type main sequence star. Although UV Aur has been known and observed as a variable star for about 60 years (MULLLER and HARTWIG 1918; ZACHAROV 1951), the photometric data obtained with the scanner from December 1970 through May 1971 shown in Figure 5 are the first to yield undiluted light curves in two colors and of photoelectric accuracy. During the period of observation, beginning well after the time of minimum light, the light curve in V showed a brightness increase of $2.2 \mathrm{mag}$ to a maximum that occurred on or about April 20, while the brightness in B rose by $3.5 \mathrm{mag}$ and reached its maximum near the end of May. The large brightness increase in $B$ compared to that in $V$ and the delay in reaching maximum light are, of course, attributable to several strong emission lines, notably $\mathrm{H} \beta$ and $\mathrm{H} \gamma$, that usually appear after minimum in the spectra of stars of this type and which continue to dominate the blue region of the spectrum well beyond the time of maximum in the V light curve. That is was, in fact, the case was confirmed by several spectra of UV Aur obtained during the 1970/71 observing season. Spectra of the blue companion of UV Aur, as 


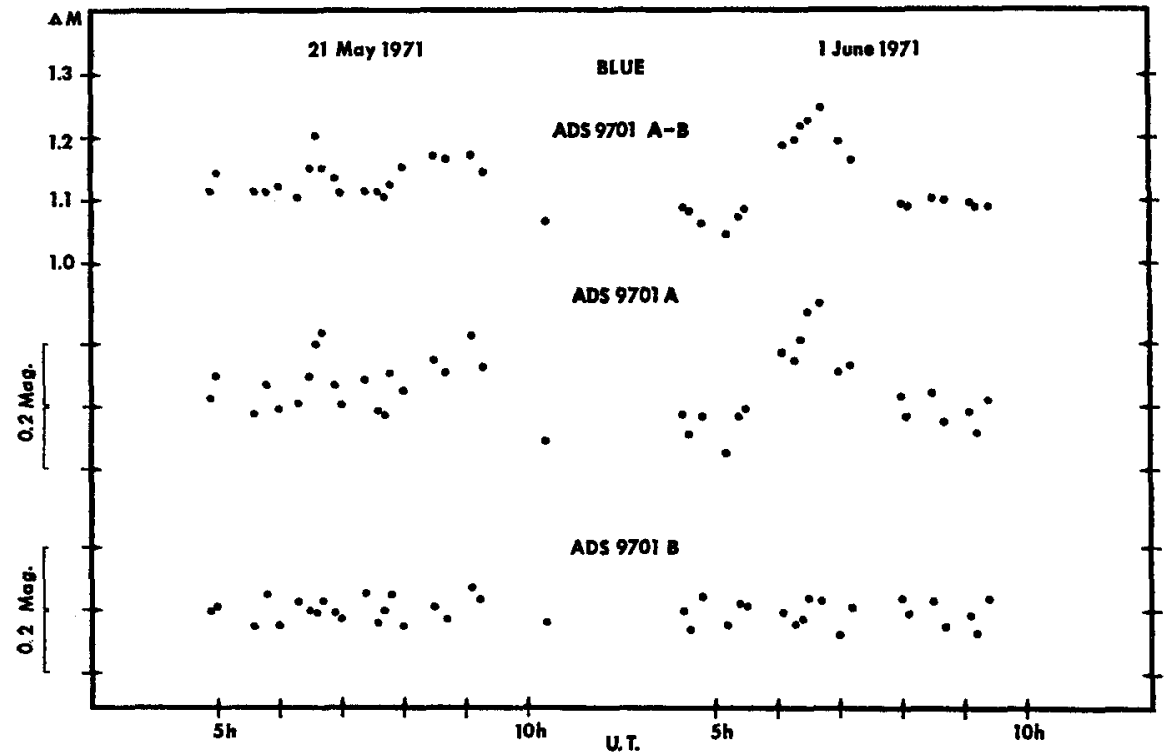

Fig. 3: "Small amplitude" (21. May 1971) and „large amplitude“ (1. June 1971) light curves of ADS $9701=\delta$ Ser. Comparison of magnitude differences between the components with instrumental magnitudes of each shows the entire variation to be due to the brighter component.

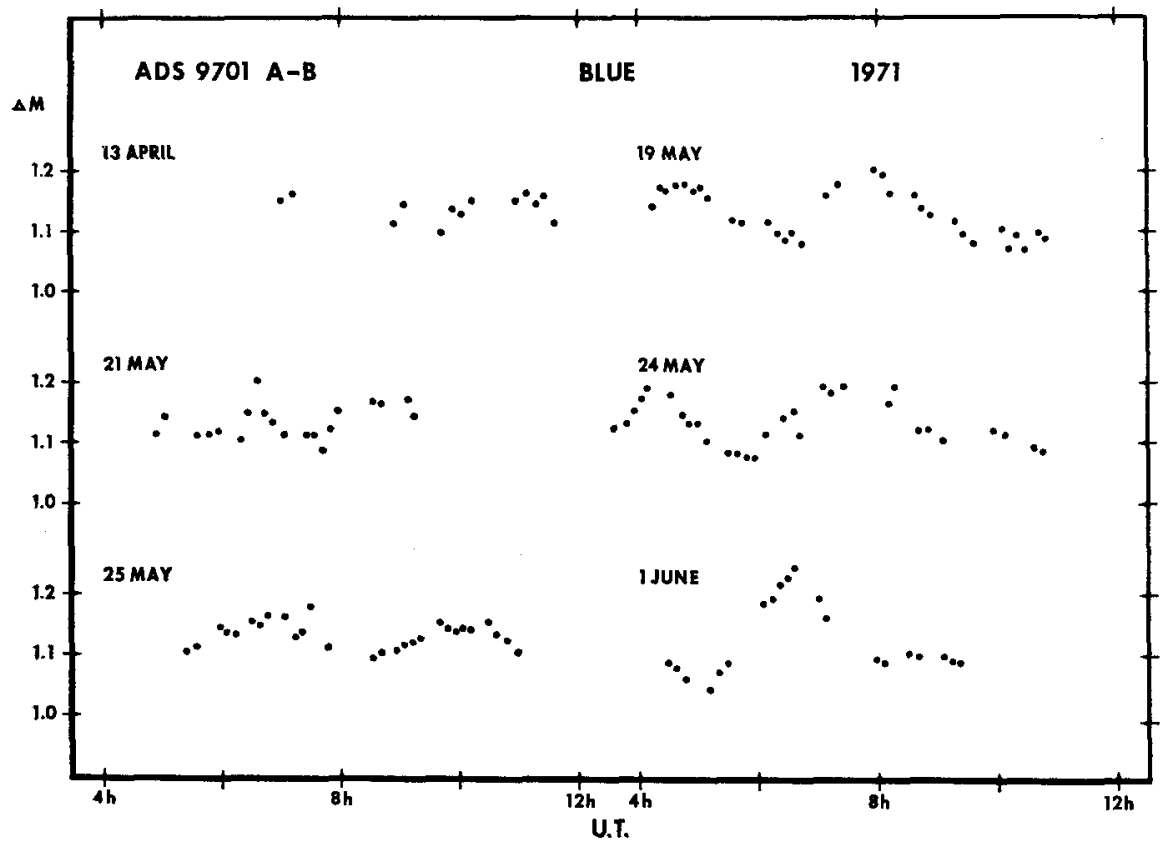

Fig. 4 : Representative light curves of $\operatorname{ADS} 9701=\delta$ Ser, showing different shapes and amplitudes ranging from less than $0.07 \mathrm{mag}$ to $0.2 \mathrm{mag}$. 


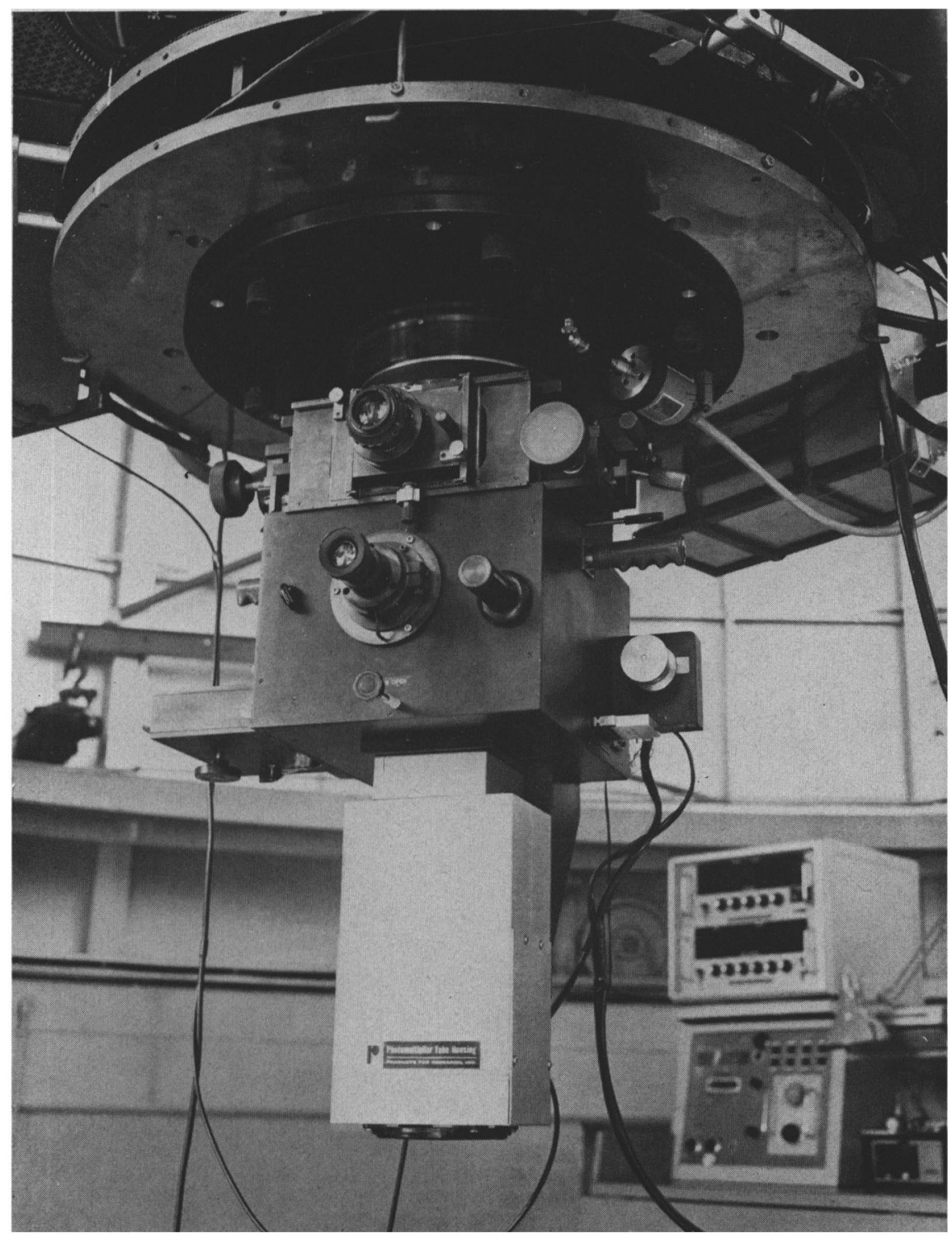

Fig. 1: Photoelectric area scanner for observation of visual double stars. 


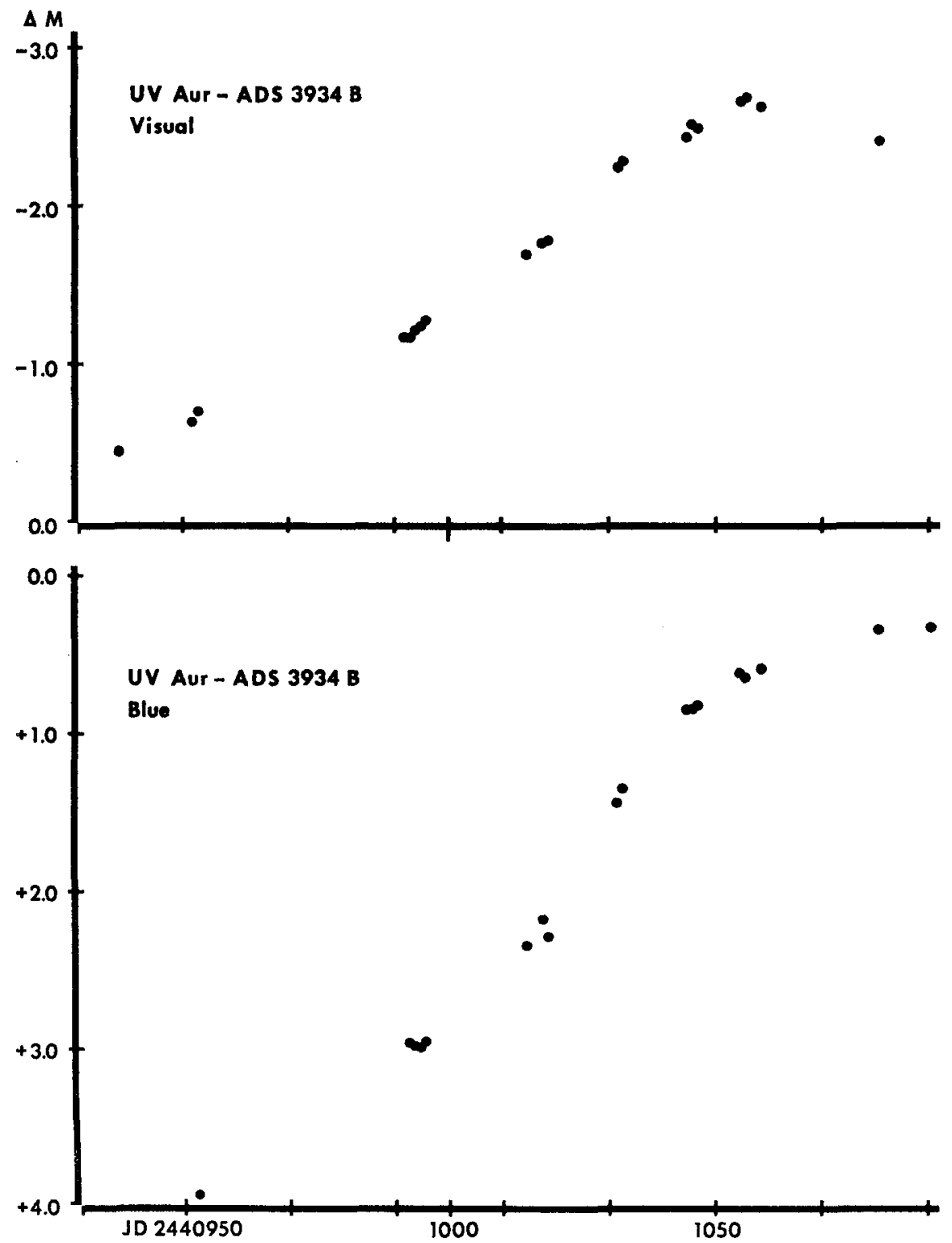

Fig. 5: Visual and blue light curves of the Mira-type carbon star UV Aur, visual primary of the 3.5-arc-sec double star ADS 3934. 
well as photoelectric observations of the combined light in the infrared, where the contribution of the blue component is negligible, were also obtained.

These new observations along with future data of the same type will lead to a significant improvement in our knowledge of the brightness variations of UV Aur. More importantly, however, our photometry and spectroscopy of both components, in conjunction with existing evidence for the physical nature of the pair, will yield a luminosity for UV Aur that may well be the best obtainable for any carbon star.

In conclusion, we believe that the two examples which we have presented here demonstrate that area scanning is a powerful technique for the study of variables in close double stars. We are currently using our instrument to obtain undiluted photometry for a wide variety of variable stars in both physical and optical doubles.

This work has been funded largely by the National Science Foundation.

$$
\text { References: }
$$

FRANZ, O. G., 1966, Lowell Obs. Bull. No 134, 251.

FRANZ, O. G., 1970, Lowell Obs. Bull. No. 154, 191.

FRANZ, O. G., and CROWFOOT, N. C., 1971, Lowell Obs. Bull. (in preparation).

MILLIS, R. L., 1967, Publ. Astron. Soc. Pacific 79, 262.

MULLER, G., and HARTWIG, E., 1918, Geschichte und Literatur des Lichtwechsels 1, 143.

RAKOS, K. D., 1965, Appl. Opt. 4, 1453 = Lowell Obs. Repr., Ser. II, No. 82.

ZACHAROV, G. P., 1951, Peremennje Zvjozdy, Bull. 8, 369.

\section{Discussion to the paper of FRANZ, MILLIS and WHITE}

SINVHAL: How do you choose the slit width in relation to the separation between the components?

WHITE: We have slit widths ranging in size from 25 to 200 microns. In practice slit widths of about $1 / 2$ second of arc are used.

QUESTER: What is the velocity/frequency of the slit crossing both components?

WHITE: Scan rates of $2.5,5$ and 10 scans per second are possible.

MAMMANO: What is the separation of the double containing UV Aur? Spectra of the star have been taken at Asiago during the recent year.

WHITE: The separation is 3.5 arc seconds.

DALLAPORTA: What is the absolute magnitude of the $\mathrm{N}$ star in UV Aur, assuming as known the absolute magnitude of the companion?

WHITE: We are in the process of studying this system and the results will be published. As of now the reductions are not final and I will only say that the companion is about a B 8 V star of apparent visual magnitude about 12.

RAKOS: I have here at the observatory my Area Scanner and I would be very glad to demonstrate it this evening. 